\title{
Deformation quantization of Poisson manifolds in the derivative expansion.
}

\author{
A.V.Bratchikov \\ Kuban State Technological University, \\ 2 Moskovskaya Street, Krasnodar, 350072, Russia
}

\begin{abstract}
Deformation quantization of Poisson manifolds is studied within the framework of an expansion in powers of derivatives of Poisson structures. We construct the Lie group associated with a Poisson bracket algebra which defines a second order deformation in the derivative expansion.
\end{abstract}

PACS 2.40 Gh

Key words: deformation quantization; non-commutative geometry.

\section{Introduction}

Let $X$ be a phase space with the phase variables $x^{i}, i=1, \ldots, N$, and the Poisson bracket

$$
\left\{x^{i}, x^{j}\right\}=\omega^{i j}(x) .
$$

Let $\tilde{A}=A[[t]]$ be the space of formal power series in a variable $t$ with coefficients in $A=C^{\infty}(X)$. A star product is a $R[[t]]$ linear associative product on $\tilde{A}$, defined for $f, g \in A$ by

$$
f * g=g f+\sum_{k=1}^{\infty} t^{k} c_{k}(f, g),
$$

where $c_{k}(f, g)$ are bidifferential operators. 
The star product is a basic object of the deformation quantization [1]. A formula for the star product on an arbitrary Poisson manifold was found by Kontsevich [2]. For symplectic Poisson manifolds one can also use Fedosov's construction of deformation quantization [3].

The star product for linear Poisson structures [4] can be derived from the Baker-Campbell-Hausdorff $(\mathrm{BCH})$ formula [5]. It is different from Kontsevich's product being a part of the last one.

In the present paper an expansion of the star product in powers of the derivatives of Poisson structure is studied. We find the explicit expression which defines a second order deformation in the derivative expansion.This expression is given by an infinite series in variable $t$ as well as in the number of first order derivatives of $\omega^{i j}$. It is based on the $\mathrm{BCH}$ formula for the Poisson bracket algebra (11).

The paper is organized as follows. In the next section we introduce notations and rewrite the associativity equation in the form which is convenient for our purposes. In Sec.3 we define an infinite-dimensional Lie group associated with the Poisson bracket algebra (1). In Sec. 4 we show that the corresponding $\mathrm{BCH}$ series defines a second order deformation quantization in the derivative expansion.

\section{Derivative expansion}

For any $f, g \in A$ the star product can be computed by the equation

$$
f(x) * g(x)=\left.f\left(\frac{\partial}{\partial \alpha}\right) g\left(\frac{\partial}{\partial \beta}\right) e^{Q(\alpha \cdot x, \beta \cdot x)}\right|_{\alpha=\beta=0},
$$

where $\alpha \cdot x=\alpha_{i} x^{i}, \alpha_{i} \in R$, and

$$
e^{Q(\alpha \cdot x, \beta \cdot x)}=e^{\alpha \cdot x} * e^{\beta \cdot x} .
$$

The associativity equation for the star product reads

$$
(f * g) * h=f *(g * h)
$$

for all $f, g, h \in \tilde{A}$. For $e^{\alpha \cdot x}, e^{\beta \cdot x}$ and $e^{\gamma \cdot x}$ this equation can be written in the form

$$
\left.\left.e^{Q(\alpha \cdot x, \beta \cdot x)}\right|_{x=\frac{\partial}{\partial \pi}} e^{Q(\pi \cdot x, \gamma \cdot x)}\right|_{\pi=0}=\left.\left.e^{Q(\beta \cdot x, \gamma \cdot x)}\right|_{x=\frac{\partial}{\partial \pi}} e^{Q(\alpha \cdot x, \pi \cdot x)}\right|_{\pi=0} .
$$


It is easy to see that equations (3) and (44) are equivalent:

$$
\begin{aligned}
& (f * g) * h=\left.\left.f\left(\frac{\partial}{\partial \alpha}\right) g\left(\frac{\partial}{\partial \beta}\right) h\left(\frac{\partial}{\partial \gamma}\right) e^{Q(\alpha \cdot x, \beta \cdot x)}\right|_{x=\frac{\partial}{\partial \pi}} e^{Q(\pi \cdot x, \gamma \cdot x)}\right|_{\pi=\alpha=\beta=\gamma=0}= \\
& =\left.\left.f\left(\frac{\partial}{\partial \alpha}\right) g\left(\frac{\partial}{\partial \beta}\right) h\left(\frac{\partial}{\partial \gamma}\right) e^{Q(\beta \cdot x, \gamma \cdot x)}\right|_{x=\frac{\partial}{\partial \pi}} e^{Q(\alpha \cdot x, \pi \cdot x)}\right|_{\pi=\alpha=\beta=\gamma=0}=f *(g * h) .
\end{aligned}
$$

Let $B$ be the algebra with respect to the pointwise multiplication generated by $\omega$ and its derivatives. Let $B_{n}, n \in N$, be the ideal of $B$ generated by $n$-th and higher derivatives of $\omega$ and $\tilde{B}_{n}=B / B_{n}$. When associativity equation (4) holds on $\tilde{B}_{n}$ we say that product (2) defines an $n$-th order deformation of $A$ in the derivative expansion. If $\psi-\varphi \in B_{n}$ we write $\psi=\varphi+O\left(\partial^{n} \omega\right)$.

We are interested in the expansion of $Q(\alpha \cdot x, \beta \cdot x) \in B$ in powers of derivatives of the Poisson structure $\omega=\left(\omega^{i j}\right)$

$$
Q(\alpha \cdot x, \beta \cdot x)=(\alpha+\beta) \cdot x+\sum_{n=1}^{\infty} Q_{n}(\alpha \cdot x, \beta \cdot x),
$$

where $Q_{n} \in B_{n-1}, Q_{n} \notin B_{n}$. One checks that

$$
Q_{1}(\alpha \cdot x, \beta \cdot x)=\frac{t}{2} \alpha_{i} \omega^{i j}(x) \beta_{j}
$$

defines a first order deformation in the derivative expansion.

\section{Lie groups associated with Poisson bracket algebras}

Let $V^{n}=V \otimes V \otimes \ldots \otimes V$ ( $n$ copies $), V^{0}=R$, be the space over $R$ which is generated by

$$
x^{i_{1}} \otimes x^{i_{2}} \otimes \ldots \otimes x^{i_{n}} .
$$

Let $K$ denote the tensor algebra

$$
K=\bigoplus_{n=0}^{\infty} V^{n}
$$


One can make $K$ into a Lie algebra $K$ by taking as Lie product $[a, b]=$ $a \otimes b-b \otimes a$. The Lie elements

$$
\left[x^{i_{n}}, \ldots,\left[x^{i_{2}}, x^{i_{1}}\right]\right]
$$

generate a Lie subalgebra $L$ of $K$.

Let $H(.,$.$) denote the \mathrm{BCH}$ series in $L$

$$
H(a, b)=a+b+\frac{1}{2}[a, b]+\frac{1}{12}[a,[a, b]]+\frac{1}{12}[b,[b, a]]+\ldots
$$

The function $H(.,$.$) satisfies the equations (see e.g. [6])$

$$
\begin{gathered}
H(a, 0)=H(0, a)=a, \quad H(a,-a)=0, \\
H(H(a, b), c)=H(a, H(b, c)) \quad \text { for all } a, b, c \in L .
\end{gathered}
$$

Let $T: L \rightarrow \tilde{A}$ be the linear function which is defined by

$$
T\left(\left[x^{i_{n}}, \ldots,\left[x^{i_{2}}, x^{i_{1}}\right]\right]\right)=t^{n-1}\left\{x^{i_{n}}, \ldots,\left\{x^{i_{2}}, x^{i_{1}}\right\}\right\}
$$

where

$$
\{f(x), g(x)\}=\partial_{i} f(x) \omega^{i j}(x) \partial_{j} g(x)
$$

and $\partial_{i}=\partial / \partial x^{i}$. The following lemma is easily proved by induction.

LEMMA The map $T$ is a homomorphism of Lie algebras.

The homomorphic images of equations (5) in $\tilde{A}$ reads

$$
\begin{gathered}
H^{\prime}\left(a^{\prime}, 0\right)=H^{\prime}\left(0, a^{\prime}\right)=a^{\prime}, \quad H^{\prime}\left(a^{\prime},-a^{\prime}\right)=0, \\
H^{\prime}\left(H^{\prime}\left(a^{\prime}, b^{\prime}\right), c^{\prime}\right)=H^{\prime}\left(a^{\prime}, H^{\prime}\left(b^{\prime}, c^{\prime}\right)\right)
\end{gathered}
$$

where $a^{\prime}=T(a), b^{\prime}=T(b), c^{\prime}=T(c)$ and $H^{\prime}(.,$.$) is the BCH series for the$ Poisson bracket $t\{.,$.

$$
H^{\prime}(f, g)=f+g+\frac{t}{2}\{f, g\}+\frac{t^{2}}{12}\{f,\{f, g\}\}+\frac{t^{2}}{12}\{g,\{g, f\}\}+\ldots
$$

Equations (6) enables us to define the Lie group $G$ which is generated by the functions $e^{\alpha \cdot x}$ and the product

$$
e^{\alpha \cdot x} \circ e^{\beta \cdot x}=e^{H^{\prime}(\alpha \cdot x, \beta \cdot x)}
$$


Each element of $G$ can be written in the form

$$
\prod_{i=1}^{s}\left(e^{\gamma^{(i)} \cdot x} \circ\right)=e^{\sum_{i=1}^{s} \gamma^{(i)} \cdot x}(1+O(t))
$$

for some values of $s$ and group parameters $\left(\gamma^{(1)}, \gamma^{(2)}, \ldots, \gamma^{(s)}\right)$. Here

$$
\prod_{i=1}^{s}\left(e^{\gamma^{(i)} \cdot x} \circ\right)=e^{\gamma^{(1)} \cdot x} \circ e^{\gamma^{(2)} \cdot x} \circ \ldots \circ e^{\gamma^{(s)} \cdot x} .
$$

It is easy to see by making use of (7) that

$$
G^{t}=\{u \in G \mid u=1+O(t)\}
$$

is a normal subgroup of $G$ and $G / G^{t}$ is isomorphic to the translation group $\hat{R}^{N}=\left\{\chi_{\alpha} \mid \chi_{\alpha}=e^{\alpha \cdot x}\right\}$. Therefore $G$ can be treated as a deformation of $\hat{R}^{N}$.

\section{A second order deformation in the deriva- tive expansion}

Let us define the $R[[t]]$ linear product on $\tilde{A}$

$$
f(x) \diamond g(x)=\left.f\left(\frac{\partial}{\partial \alpha}\right) g\left(\frac{\partial}{\partial \beta}\right) e^{H^{\prime}(\alpha \cdot x, \beta \cdot x)}\right|_{\alpha=\beta=0 .} .
$$

It can be also written in the form

$$
f(x) \diamond g(x)=\left.e^{M\left(\frac{\partial}{\partial \xi} \cdot x, \frac{\partial}{\partial \eta} \cdot x\right)} f(\xi) g(\eta)\right|_{\xi=\eta=x},
$$

where

$$
M(f, g)=H^{\prime}(f, g)-f-g
$$

For the Poisson bracket with constant $\omega^{i j}$

$$
M\left(\frac{\partial}{\partial \xi} \cdot x, \frac{\partial}{\partial \eta} \cdot x\right)=\frac{t}{2} \omega^{i j} \frac{\partial}{\partial \xi^{i}} \frac{\partial}{\partial \eta^{j}} .
$$

In this case (8) reproduces the Groenewold-Moyal product

$$
f(x) \diamond g(x)=\left.e^{\frac{t}{2} \omega^{i j} \frac{\partial}{\partial \xi^{i}} \frac{\partial}{\partial \eta^{j}}} f(\xi) g(\eta)\right|_{\xi=\eta=x} .
$$


Product (8) coincides also with the star product for linear Poisson structures [5].

Expanding (8) in powers of $t$ one gets

$$
\begin{aligned}
f \diamond g=f g+\frac{t}{2} \omega^{i j} \partial_{i} f \partial_{j} g+ & \frac{t^{2}}{8} \omega^{i j} \omega^{k l} \partial_{i} \partial_{k} f \partial_{j} \partial_{l} g+ \\
& +\frac{t^{2}}{12} \omega^{i j} \partial_{j} \omega^{k l}\left(\partial_{i} \partial_{k} f \partial_{l} g-\partial_{k} f \partial_{i} \partial_{l} g\right)+O\left(t^{3}\right) .
\end{aligned}
$$

This means that (8) defines a second order deformation in the variable $t$ [2, 7].

THEOREM Formula (8) gives a second order deformation of $A$ in the derivative expansion.

Proof. Let $l(x)$ be a Lie series in $x^{i}$. It can be written as

$$
l(x)=\alpha \cdot x+\sum_{n=2}^{\infty} \alpha_{i_{1} i_{2} \ldots i_{n}}\left\{x^{i_{n}}, \ldots,\left\{x^{i_{2}}, x^{i_{1}}\right\}\right\}
$$

for some $\alpha_{i_{1} i_{2} \ldots i_{n}} \in R$. One checks that

$$
l(x+a)=l(x)+\partial_{i} l(x) a^{i}+O\left(\partial^{2} \omega\right)
$$

and for any function $f(x)$

$$
\{l(x), f(x)\}=\left\{\partial_{i} l(x) x^{i}, f(x)\right\}+O\left(\partial^{2} \omega\right) .
$$

From this it follows

$$
H^{\prime}(l(x), \gamma \cdot x)=l(x)+\gamma \cdot x+M\left(\partial_{i} l(x) x^{i}, \gamma \cdot x\right)+O\left(\partial^{2} \omega\right) \text {. }
$$

Let us compute the left-hand side of equation (4) for $Q(\alpha \cdot x, \beta \cdot x)=$ $H^{\prime}(\alpha \cdot x, \beta \cdot x)$. Using (9) and (11) one gets

$$
\begin{aligned}
& \left.\left.e^{H^{\prime}(\alpha \cdot x, \beta \cdot x)}\right|_{x=\frac{\partial}{\partial \pi}} e^{H^{\prime}(\pi \cdot x, \gamma \cdot x)}\right|_{\pi=0}=\left.\left.e^{H^{\prime}(\alpha \cdot x, \beta \cdot x)}\right|_{x=x+\frac{\partial}{\partial \pi}} e^{\gamma \cdot x+M(\pi \cdot x, \gamma \cdot x)}\right|_{\pi=0}= \\
= & e^{\gamma \cdot x+H^{\prime}(\alpha \cdot x, \beta \cdot x)+M\left(\partial_{i} H^{\prime}(\alpha \cdot x, \beta \cdot x) x^{i}, \gamma \cdot x\right)}+O\left(\partial^{2} \omega\right)=e^{\left.H^{\prime}\left(H^{\prime}(\alpha \cdot x, \beta \cdot x), \gamma \cdot x\right)\right)}+O\left(\partial^{2} \omega\right) .
\end{aligned}
$$

By similar computations of the right-hand side of (4) we obtain

$$
\left.\left.e^{H^{\prime}(\beta \cdot x, \gamma \cdot x)}\right|_{x=\frac{\partial}{\partial \pi}} e^{H^{\prime}(\alpha \cdot x, \pi \cdot x)}\right|_{\pi=0}=e^{H^{\prime}\left(\alpha \cdot x, H^{\prime}(\beta \cdot x, \gamma \cdot x)\right)}+O\left(\partial^{2} \omega\right) .
$$


From (12), (13) and (6) it follows that $H^{\prime}(\alpha \cdot x, \beta \cdot x)$ satisfies equation (41) modulo $O\left(\partial^{2} \omega\right)$. This means that

$$
(f \diamond g) \diamond h=f \diamond(g \diamond h)+O\left(\partial^{2} \omega\right)
$$

for all $f, g, h \in \tilde{A}$.

\section{Conclusion}

In the present article we have constructed the Lie group associated with a Poisson bracket algebra. We have found that the corresponding $\mathrm{BCH}$ series defines a second order deformation of Poisson manifolds in the derivative expansion.

It would be interesting to find a third order deformation. It defines a deformation of quadratic Poisson structures.

\section{References}

[1] F.Bayen,M.Flato,C.Fronsdal,A.Lichnerovich,D.Sternheimer, Ann.Phys. 111 (1978) 61 .

[2] M.Kontsevich, Lett.Math.Phys. 66 (2003) 157.

[3] B.Fedosov, J.Diff.Geom. 40 (1994) 213.

[4] S.Gutt, Lett.Math.Phys.7 (1983) 249 .

[5] V.Kathotia, Kontsevich's universal formula for deformation quantization and the Campbell-Baker-Hausdorff formula,I, math.QA/9811174.

[6] N.Bourbaki, Groupes et algebres de Lie (Hermann,Paris, 1971,1972).

[7] M.Penkava, P.Vanhaecke, Deformation quantization of polynomial Poisson algebras, math.QA/9804022. 